\title{
Dynamic Adaptive Streaming over HTTP (DASH) within P2P systems: a survey

\author{
Koffka Khan
}

Department of Computing and Information Technology the University of the West Indies, Trinidad and Tobago, W.I Email: koffka.khan@gmail.com

\section{Wayne Goodridge}

Department of Computing and Information Technology The University of the West Indies, Trinidad and Tobago, W.I

Email: wayne.goodridge@sta.uwi.edu.com

-ABSTRACT-

Video-over-IP applications have recently attracted a large number of users on the Internet. Traditional clientserver based video streaming solutions incur lavish bandwidth provision cost on the server. Peer-to-Peer (P2P) networking is a new paradigm to build distributed network applications. Recently, several P2P streaming systems have been deployed to provide live and on-demand video streaming services on the Internet at low server cost. This paper explores dynamic adaptive streaming over HTTP (DASH) within P2P systems.

Keywords - Video-over-IP; Internet; Peer-to-Peer; P2P; streaming; DASH.

Date of Acceptance: July 20, 2019

\section{INTRODUCTION}

$\mathrm{P}$ new paradigm to build distributed network applications. The basic design philosophy of P2P is to encourage users to act as both clients and servers, namely as peers. In a P2P network, a peer not only downloads data from the network, but also uploads the downloaded data to other users in the network. The uploading bandwidth of end users is efficiently utilized to reduce the bandwidth burdens otherwise placed on the servers. P2P file sharing applications, have been widely employed to quickly disseminate data files on the Internet [2]. More recently, P2P technology has been employed to provide media streaming services [17], [15]. Several P2P streaming systems have been deployed to provide on-demand or live video streaming services over the Internet.

Streaming audio and video content currently accounts for the majority of the internet traffic and is typically deployed over the top of the existing infrastructure. We are facing the challenge of a plethora of media players and adaptation algorithms. With companies such as Netflix and YouTube accounting for more than $50 \%$ of the peak download traffic on North American fixed networks in 2015 video streaming represents a significant source of Internet traffic [10]. Dynamic adaptive streaming over HTTP (DASH) has recently emerged as a standard for Internet video streaming. A range of rate adaptation mechanisms are proposed for DASH systems in order to deliver video quality that matches the throughput of dynamic network conditions for a richer user experience.

This work consists of four sections. Section II presents a categorization of DASH within P2P systems. Section III gives examples of DASH within P2P systems. Finally, the conclusion is given in Section IV.

\section{DASH WITHIN P2P SYSTEMS}

DASH within P2P systems are categorized into Overlay Nets, New Media, Collaborative, Multi-Source, Multicast,
Switching, Caching and Incentive-based (see Figure 1). New Media is broken down into Multiview and 3D. Collaborative is broken down into Bottleneck Management. Finally, Pull-based is a component of Caching.

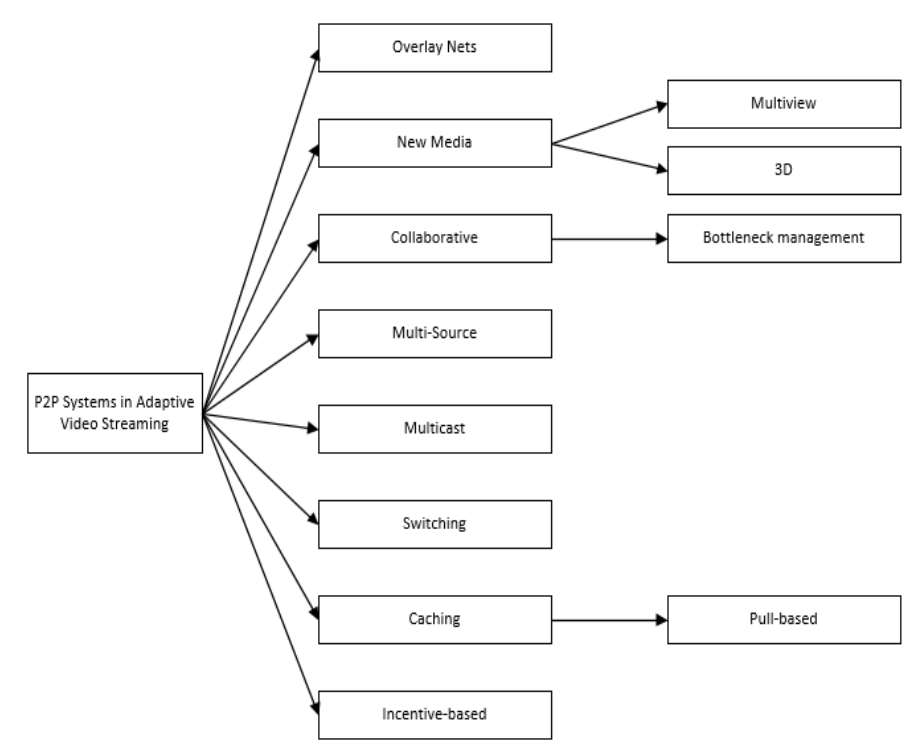

Fig. 1.DASH within P2P systems.

\section{EXAMPLES OF DASH WITHIN P2P SYSTEMS}

\section{A. Overlay Nets}

Authors present DONet, a data-driven overlay network for live media streaming [20]. The core operations in DONet are very simple: every node periodically exchanges data availability information with a set of partners, and retrieves unavailable data from one or more partners, or supplies available data to partners. Authors emphasize three salient features of this data-driven design: 1) easy to 
implement, as it does not have to construct and maintain a complex global structure; 2) efficient, as data forwarding is dynamically determined according to data availability while not restricted by specific directions; and 3) robust and resilient, as the partnerships enable adaptive and quick switching among multi-suppliers. Authors show through analysis that DONet is scalable with bounded delay. They also address a set of practical challenges for realizing DONet, and propose an efficient member and partnership management algorithm, together with an intelligent scheduling algorithm that achieves real-time and continuous distribution of streaming contents. Authors have extensively evaluated the performance of DONet over the PlanetLab. Their experiments, involving almost all the active PlanetLab nodes, demonstrate that DONet achieves quite good streaming quality even under formidable network conditions. Moreover, its control overhead and transmission delay are both kept at low levels. An Internet-based DONet implementation, called CoolStreaming v.0.9, was released on May 30, 2004, which has attracted over 30000 distinct users with more than 4000 simultaneously being online at some peak times. Authors discuss the key issues toward designing CoolStreaming in this paper, and present several interesting observations from these large-scale tests; in particular, the larger the overlay size, the better the streaming quality it can deliver.

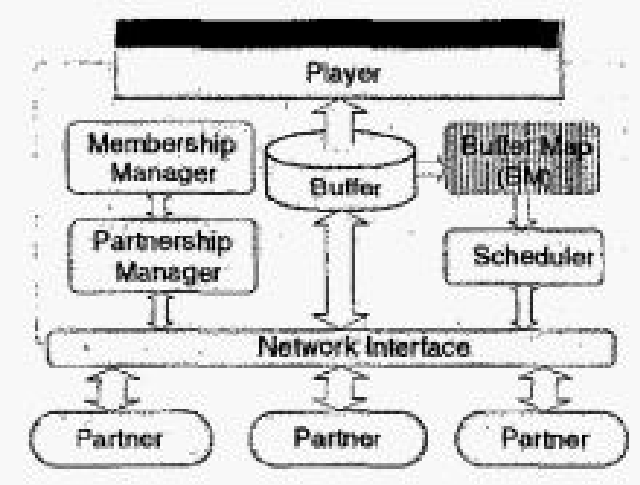

Fig. 2. A generic system diagram for a DONet node [20].

\section{B. New Media}

\section{Multiview}

Multiview entertainment is the next step in 3D immersive media networking owing to its improved depth perception and free-viewpoint viewing capability whereby users can observe the scene from the desired viewpoint. Authors outline a delivery system for multiview plus depth video, combining the broadcast and broadband networks [1]. The digital video broadcast (DVB) network is used along with adaptive peer-to-peer (P2P) distribution over the Internet to deliver high-volume multimedia to users. The DVB network has been used to deliver part of the 3D service, owing to its robustness and wide availability, as a mechanism to guarantee the minimum 3D quality of experience. The developed system brings key contributions in the $\mathrm{P} 2 \mathrm{P}$ transport for real-time multimedia delivery, including a user preference-aware adaptation mechanism, adaptive redundant chunk scheduling for robustness, incentives to decrease the load on the content server for improved system scalability, and resynchronization capability with the DVB transmission. The introduced features are compared with those of some other well-known P2P solutions to highlight the quantitative gains. A subjective testing campaign has also been organized on the developed hybrid platform, which proves the effectiveness of user-aware adaptation over network-based adaptation on a mean opinion score scale.

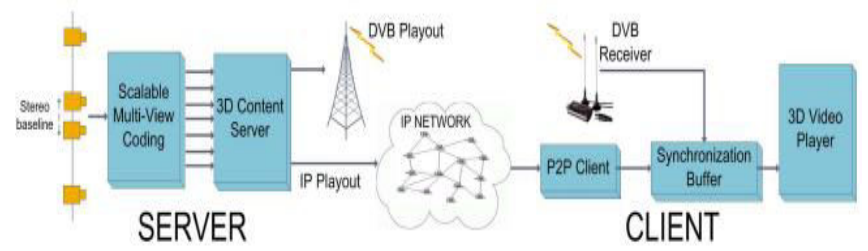

Fig. 3. Overview of the hybrid MVV content delivery architecture [1].

2. $3 D$

Peer-to-peer (P2P) systems are becoming increasingly popular due to their ability to deliver large amounts of data at a reduced deployment cost. This becomes especially useful in rendering 3D applications. In addition to fostering the development of novel media applications, $\mathrm{P} 2 \mathrm{P}$ systems also represent an interesting alternative paradigm for media streaming applications that can benefit from the inherent self-organization and resource scalability available in such environments. Authors present an overview of application and network layer mechanisms that enable successful streaming frameworks in peer-topeer systems [3]. They describe media delivery architectures that can be deployed over P2P networks to address the specific requirements of streaming applications. In particular, authors show how videostreaming applications can benefit from the diversity offered by P2P systems and implement distributedstreaming and scheduling solutions with multi-path [5] packet transmission.

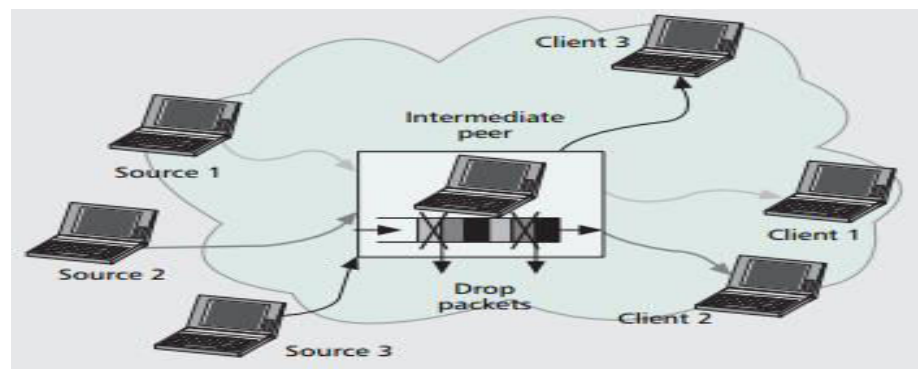

Fig. 4.Intermediate peer processing of the incoming media streams. Based on distortion information present in the packet headers, a node can make a rate-distortion optimized scheduling decision for the incoming packets [3].

\section{Collaborative}

Authors present a new framework for Peer-to-Peer Adaptive Layered Streaming, called PALS [13]. PALS is a 
receiver-driven approach for quality adaptive playback of layer encoded streaming media from a group of congestion controlled sender peers to a single receiver peer. Since the effective throughput from each sender is variable and not known a priori, it is challenging to coordinate delivery among active senders. In PALS, the receiver orchestrates coordinated delivery among active senders by adaptively determining: 1) a subset of senders that maximize overall throughput, 2) overall quality (i.e. number of layers) that can be delivered from these senders as well as distribution of overall throughput among active layers, and most importantly 3) required packets to be delivered by each active sender in order to effectively cope with any sudden change in throughput from individual senders. Authors describe PALS framework, identify key components of the framework and their interesting design challenges, present sample solution for the key components, and present their preliminary results.

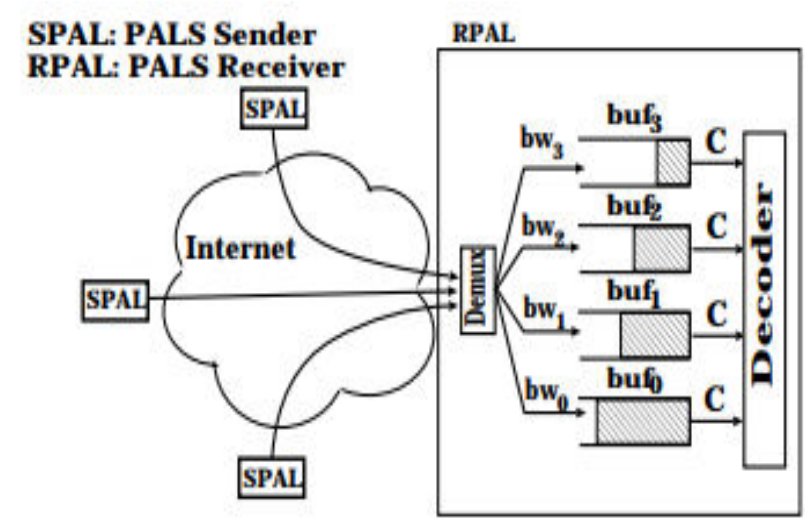

Fig. 5.End-to-end Architecture in PALS [13].

\section{Bottleneck Management}

Authors address the problem of quality bottleneck in adaptive SVC streaming [11]. Quality bottleneck occurs in adaptive streaming systems when the desired video quality cannot be obtained even if the network capabilities are sufficient. In the context of SVC layered video streaming, authors have observed that enhancement layers remain around the video source and fail to reach all the participating peers. To overcome this problem, the authors propose an adaptive SVC streaming solution that cooperatively integrates strategies of overlay formation, data scheduling and content adaptation. Performance evaluation using simulations shows that the proposed streaming solution reduces the quality bottleneck, increases churn-tolerance and optimizes bandwidth utilization.

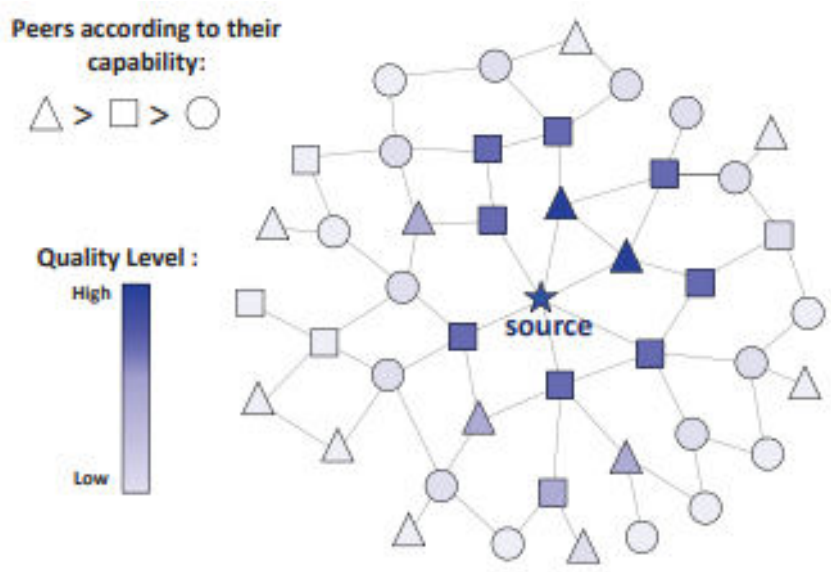

Fig. 6. Quality Bottleneck in Adaptive SVC Streaming [11].

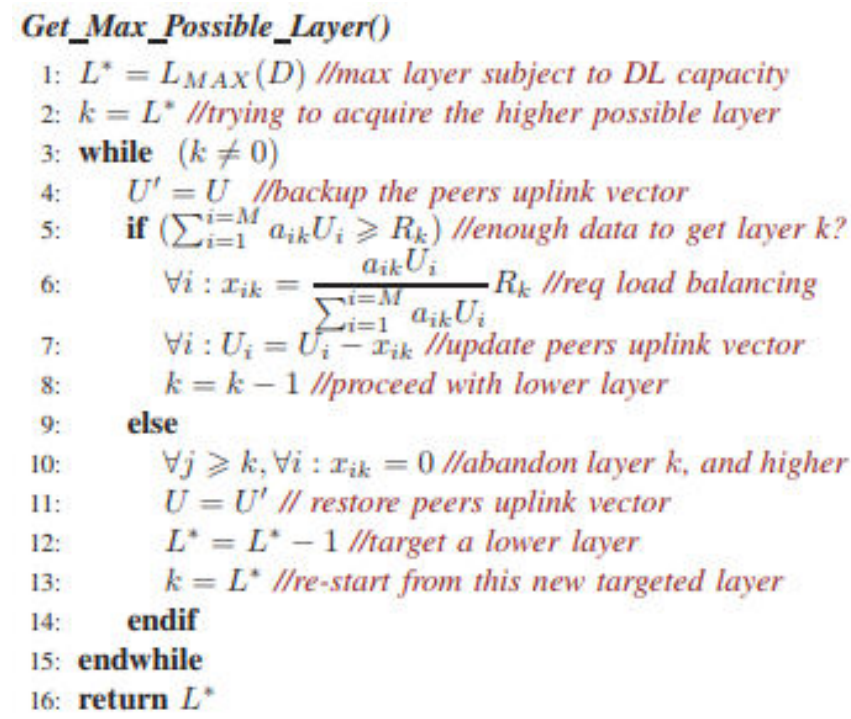

Fig. 7. Scheduling Strategy [11].

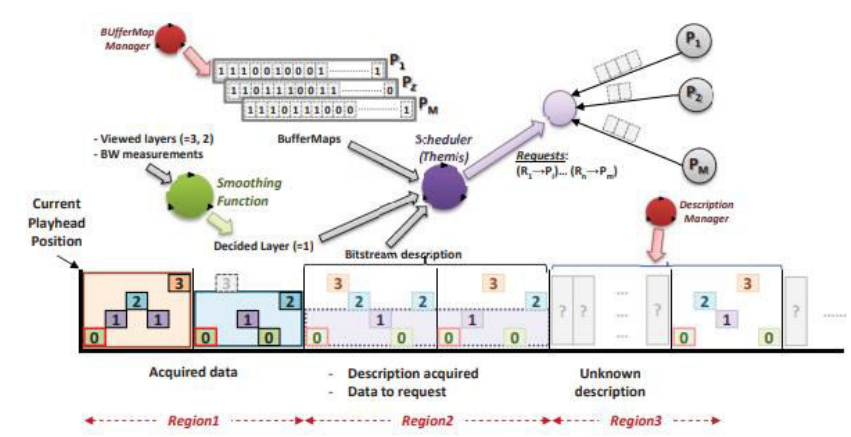

Fig. 8. Overview of the Streaming System [11].

\section{D.Multi-Source}

Authors focus on the real-time streaming of IP packet video [12]. They consider the scenario where there exist have multiple senders (or multiple sources: multi-source) that stream the same video to single receiver over Peer-toPeer networks. Authors propose an adaptive scheme for video streaming over $\mathrm{P} 2 \mathrm{P}$ network that encompasses an efficient mechanism for the selection and the maintenance 
of sender peers nodes. Furthermore, authors perform active measurements of links between the receiver and stream senders in order to optimize the overall video quality. Finally, the evaluation conducted over ns2 simulations shows that our solution allows to efficiently utilize available network bandwidth of sending peers and allow maximizing streaming qualities at the reception peer.

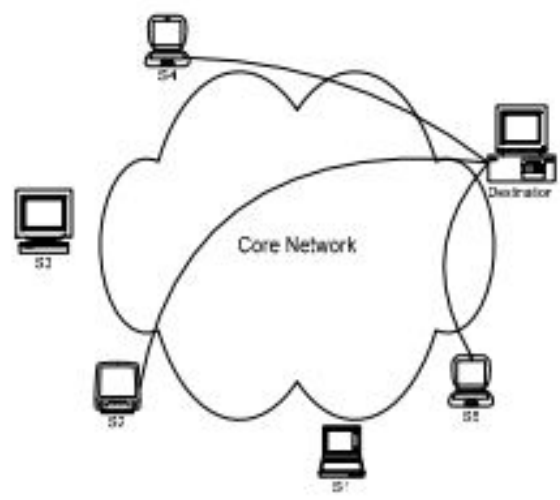

Fig. 9. Target Architecture (Many senders toward one receiver) [12].

\section{E. Multicast}

Authors study peer-to-peer multicast streaming, where a source distributes real-time video to a large population of hosts by making use of their forwarding capacity rather than relying on dedicated media servers [14]. They present a distributed streaming protocol which builds and maintains multiple multicast trees. The protocol is combined with an adaptive scheduling algorithm which ensures packets destined to a large number of peers, or particularly important to decode the video, are sent in priority. Experiments carried out over a simulated network of up to 3000 peers illustrate the performance of the protocol. For low latency video streaming, the prioritization algorithm offers performance gains, especially for large audiences and low latencies.

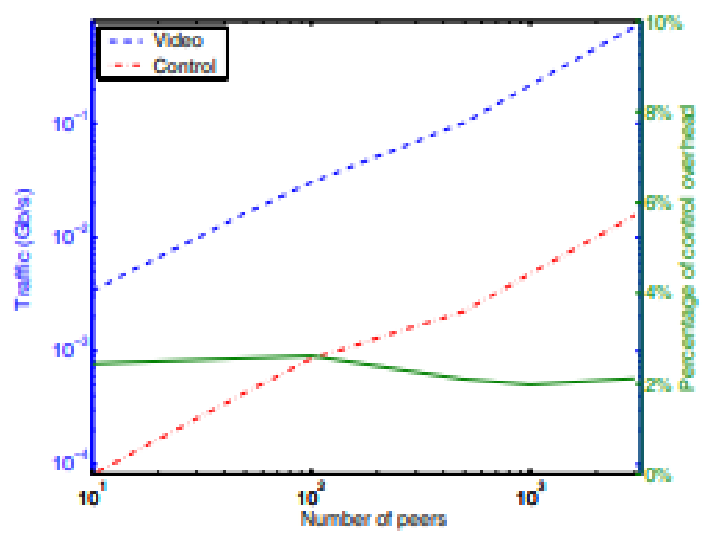

Fig. 10. Aggregate traffic and control overhead for different numbers of peers and 4 multicast trees [14].

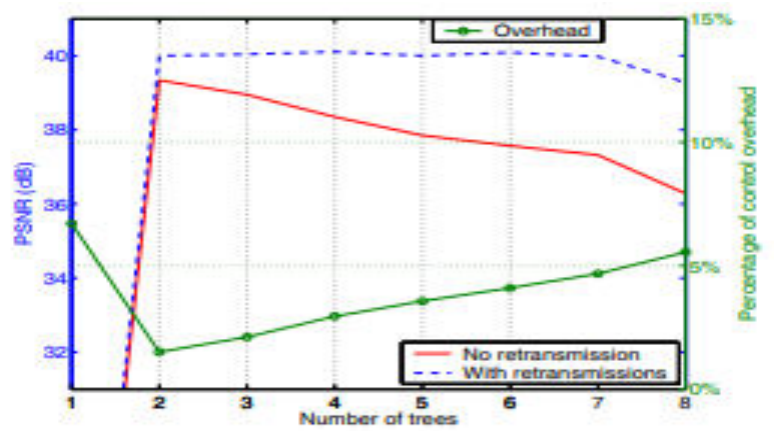

Fig. 11. Average video quality and control overhead for 300 peers and varying numbers of multicast trees [14].

\section{F. Switching}

The transmission of video content accounts for a large share of today's Internet traffic [8], [7], [6]. While Videoon-Demand $(\mathrm{VoD})$ substantially contributes to this, live streaming events such as video broadcasts from the Olympic Games can cause very high traffic volumes in the short term as well. Such peaks along with high fluctuations triggered by sudden changes in the behavior of users make the design of live streaming systems particularly challenging. Peer-to-Peer (P2P) has proven to be a scalable approach for disseminating content to a large number of users. Accordingly, the body of research offers numerous P2P live streaming approaches tailored towards specific scenarios and assumptions. However, no single approach is able to perform well under all possible conditions. Keeping up a high performance when conditions are changing is a challenge, since topology management and scheduling mechanisms cannot be exchanged easily. Therefore, authors propose TRANSIT, a new approach going beyond existing works in that it supports transitions between different live streaming mechanisms [18]. TRANSIT makes different configurations of such mechanisms seamlessly exchangeable to enable the optimal choice of configurations for a wide range of live streaming scenarios. The approach is evaluated using measurementand trace-based workloads. The results show that TRANSIT is able to maintain a high performance at a low overhead in highly fluctuating environments, whereas static configurations show serious performance degradations.

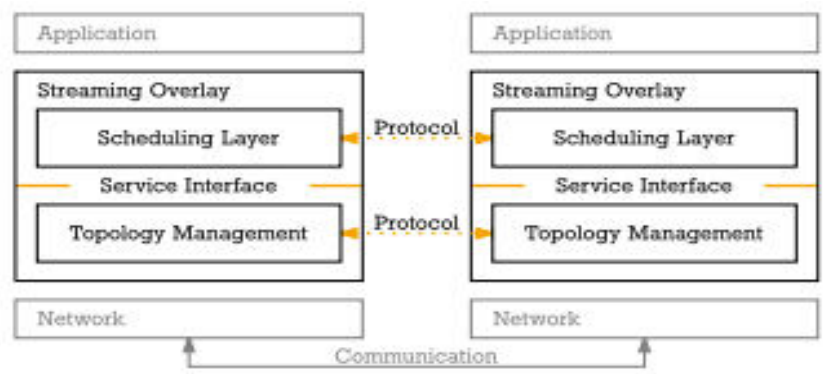

Fig. 12. System overview of TRANSIT [18]. 


\section{G. Caching}

On-demand video streaming is becoming a killer application for wireless networks. Recent informationtheoretic results have shown that a combination of caching on the users' devices and device-to-device (D2D) communications yields throughput scalability for very dense networks, which represent critical bottlenecks for conventional cellular and wireless local area network (WLAN) technologies. Authors consider the implementation of such caching D2D systems where each device pre-caches a subset of video files from a library, and users requesting a file that is not already in their library obtain it from neighboring devices through D2D communication [9]. They develop centralized and distributed algorithms for the delivery phase, encompassing a link scheduling and a streaming component. The centralized scheduling is based on the max-weighted independent set (MWIS) principle and uses message-passing to determine max-weight independent sets. The distributed scheduling is based on a variant of the FlashLinQ link scheduling algorithm, enhanced by introducing video-streaming specific weights. In both cases, the streaming component is based on a qualityaware stochastic optimization approach, reminiscent of current Dynamic Adaptive Streaming over HTTP (DASH) technology, for which users sequentially request video "chunks" by choosing adaptively their quality level. The streaming and the scheduling components are coupled by the length of the users' request queues. Through extensive system simulation, the proposed approaches are shown to provide sizeable gains with respect to baseline schemes formed by the concatenation of off-the-shelf FlashLinQ with proportional fair link scheduling and DASH at the application layer.

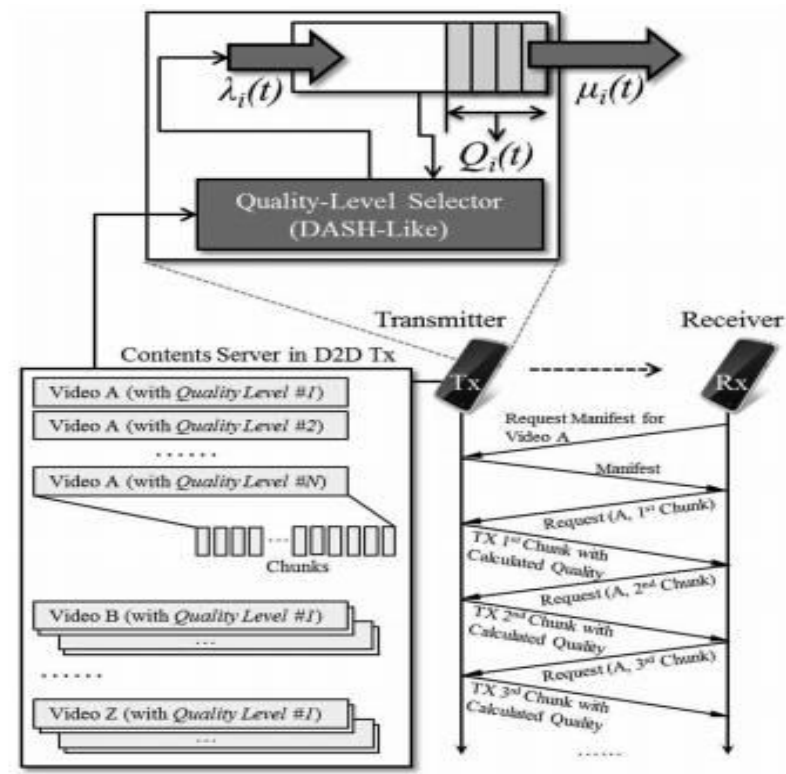

Fig. 13. Proposed DASH-like quality adaptive D2D system architecture. The D2D transmitter has its own contents server that contains various videos (with various quality levels), and each video consists of a sequence of chunks. For each D2D transmission, the transmitter can select suitable quality for each chunk transmission. The proposed algorithm can be represented as a Quality-Level Selector (DASH-Like) in this figure that determines which quality level/mode (among all possible quality levels) based on the transmitter queue backlog size [9].

\section{Pull-based}

Unlike P2P live video streaming in which all the peers in a channel watch a video with tiny differences in viewing points, in $\mathrm{P} 2 \mathrm{P}$ video on demand (VoD) streaming systems, neighbor peers may watch the same video with more different viewing points; therefore, using push-based approach is not efficient for such systems, and the overhead of the pull-based approaches is challenging due to the periodical exchange of buffer-maps among the peers. In pull-based $\mathrm{P} 2 \mathrm{P}$ VoD systems, to achieve better quality of experience it is necessary to use large buffers at the peers that results in more buffer-maps exchange overhead. Authors study buffer-map exchange challenging in pull-based P2P VoD streaming systems and propose an adaptive mechanism for decreasing overhead by sending the buffer-maps with regard to the viewing points of the peers [16]. Bandwidth overhead of the proposed mechanism is independent of the used buffer sizes and is less dependent to the buffer-map exchange period. By using this effective mechanism, better quality of service can be achieved through using large buffers at the peers, without increasing in the overhead. Authors simulation based performance evaluation show the efficiency of the proposed mechanism in decreasing the bandwidth overhead of buffer-map exchange in P2P VoD streaming systems.

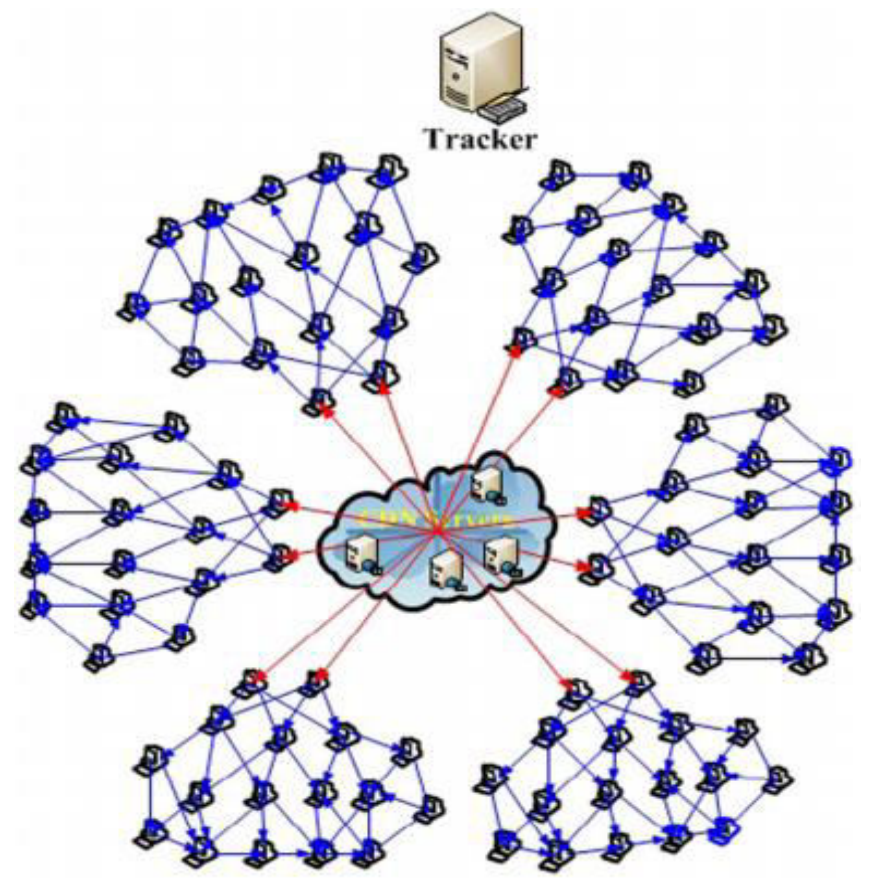

Fig. 14. Structure of designed CDN-P2P system [16]. 


\section{H.Incentive-based}

With high scalability, high video streaming quality, and low bandwidth requirement, peer-to-peer (P2P) systems have become a popular way to exchange files and deliver multimedia content over the internet. However, current P2P systems are suffering from "free-riding" due to the peers' selfish nature. Authors propose a credit-based incentive mechanism to encourage peers to cooperate with each other in a heterogeneous network consisting of wired and wireless peers [4]. The proposed mechanism can provide differentiated service to peers with different credits through biased resource allocation. A Stackelberg game is formulated to obtain the optimal pricing and purchasing strategies, which can jointly maximize the revenue of the uploader and the utilities of the downloaders. In particular, peers' heterogeneity and selfish nature are taken into consideration when designing the utility functions for the Stackelberg game. It is shown that the proposed resource allocation scheme is effective in providing service differentiation for peers and stimulating them to make contribution to the P2P streaming system.

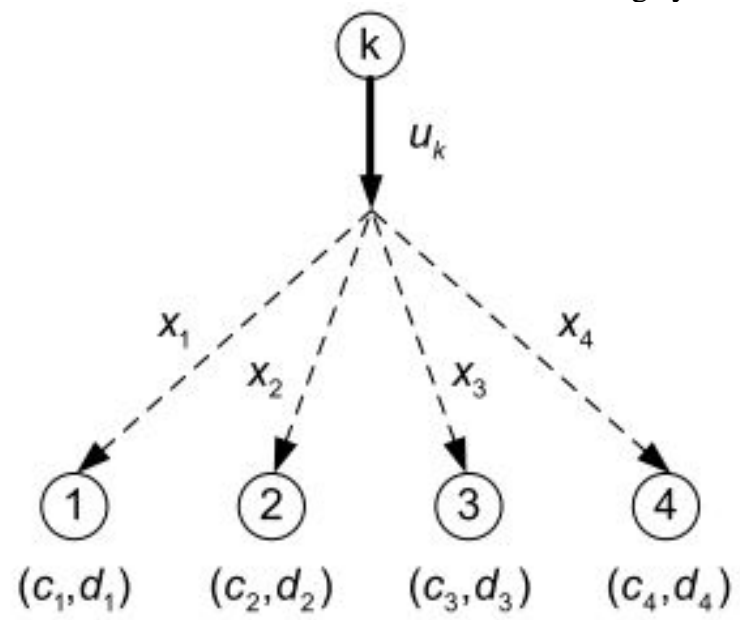

Fig. 15. System model [4].

\section{CONCLUSION}

Video-over-IP applications have recently attracted a large number of users on the Internet. Traditional client-server based video streaming solutions incur lavish bandwidth provision cost on the server. Peer-to-Peer (P2P) networking is a new paradigm to build distributed network applications. Recently, several P2P streaming systems have been deployed to provide live and on-demand video streaming services on the Internet at low server cost. This paper explored dynamic adaptive streaming over HTTP (DASH) within P2P systems.

\section{REFERENCES}

[1] Ekmekcioglu, Erhan, Cihat Goktug Gurler, Ahmet Kondoz, and Ahmet Murat Tekalp. "Adaptive multiview video delivery using hybrid networking." IEEE Transactions on Circuits and Systems for Video Technology 27, no. 6 (2017): 1313-1325.

[2] Goswami, Antriksh, Gopal Sharan Parashari, and Ruchir Gupta. "Evolutionary Stability of Reputation-
Based Incentive Mechanisms in P2P Systems." IEEE Communications Letters 22, no. 2 (2018): 268-271.

[3] Jurca, Dan, Jacob Chakareski, Jean-Paul Wagner, and Pascal Frossard. "Enabling adaptive video streaming in P2P systems [Peer-to-Peer Multimedia Streaming]." IEEE Communications Magazine 45, no. 6 (2007): 108-114.

[4] Kang, Xin, and Yongdong Wu. "Incentive mechanism design for heterogeneous peer-to-peer networks: A Stackelberg game approach." IEEE Transactions on Mobile Computing 14, no. 5 (2015): 1018-1030.

[5] Khan, Koffka, and Wayne Goodridge. "Energy aware Ad-Hoc on demand multipath distance vector routing." International Journal of Intelligent Systems and Applications 7, no. 7 (2015): 50-56.

[6] Khan, Koffka, and Wayne Goodridge. "Machine learning in Dynamic Adaptive Streaming over HTTP (DASH)." International Journal of Advanced Networking and Applications 9, no. 3 (2017): 34613468.

[7] Khan, Koffka, and Wayne Goodridge. "QoE in DASH." International Journal of Advanced Networking and Applications 9, no. 4 (2018): 35153522.

[8] Khan, Koffka, and Wayne Goodridge. "Server-based and network-assisted solutions for adaptive video streaming." International Journal of Advanced Networking and Applications 9, no. 3 (2017): 34323442.

[9] Kim, Joongheon, Giuseppe Caire, and Andreas F. Molisch. "Quality-aware streaming and scheduling for device-to-device video delivery." IEEE/ACM Transactions on Networking 24, no. 4 (2016): 23192331.

[10]Kua, Jonathan, Grenville Armitage, and Philip Branch. "A survey of rate adaptation techniques for dynamic adaptive streaming over HTTP." IEEE Communications Surveys \& Tutorials 19, no. 3 (2017): 1842-1866.

[11] Medjiah, Samir, Toufik Ahmed, and Raouf Boutaba. "Avoiding quality bottlenecks in P2P adaptive streaming." IEEE Journal on Selected Areas in Communications 32, no. 4 (2014): 734-745.

[12] Mushtaq, Mubashar, Toufik Ahmed, and DjamalEddine Meddour. "Adaptive packet video streaming over P2P networks." In Proceedings of the 1st international conference on Scalable information systems, p. 59. ACM, 2006.

[13]Rejaie, Reza, and Antonio Ortega. "PALS: peer-topeer adaptive layered streaming." In Proceedings of the 13th international workshop on Network and operating systems support for digital audio and video, pp. 153-161. ACM, 2003.

[14] Setton, Eric, Jeonghun Noh, and Bernd Girod. "Low latency video streaming over peer-to-peer networks." In Multimedia and Expo, 2006 IEEE International Conference on, pp. 569-572. IEEE, 2006.

[15] Shehab, Abdulaziz, Mohamed Elhoseny, Mohamed Abd El Aziz, and Aboul Ella Hassanien. "Efficient schemes for playout latency reduction in $\mathrm{P} 2 \mathrm{P}-\mathrm{VoD}$ 
systems." In Advances in Soft Computing and Machine Learning in Image Processing, pp. 477-495. Springer, Cham, 2018.

[16] Sheshjavani, Abdollah Ghaffari, and Behzad Akbari. "An adaptive buffer-map exchange mechanism for pull-based peer-to-peer video-on-demand streaming systems." Multimedia Tools and Applications 76, no. 5 (2017): 7535-7561.

[17] Wang, Haizhou, Xingshu Chen, Wenxian Wang, and Mei Ya Chan. "Content Pollution Propagation in the Overlay Network of Peer-to-Peer Live Streaming Systems: Modeling and Analysis." IET Communications (2018).

[18] Wichtlhuber, Matthias, Bjorn Richerzhagen, Julius Ruckert, and David Hausheer. "TRANSIT: Supporting transitions in Peer-to-Peer live video streaming." In Networking Conference, 2014 IFIP, pp. 1-9. IEEE, 2014.

[19]Zhang, Jianwei, Xinchang Zhang, and Chunling Yang. "Towards the multi-request mechanism in pullbased peer-to-peer live streaming systems." Computer Networks 138 (2018): 77-89.

[20]Zhang, Xinyan, Jiangchuan Liu, Bo Li, and Y-SP Yum. "CoolStreaming/DONet: A data-driven overlay network for peer-to-peer live media streaming." In INFOCOM 2005. 24th Annual Joint Conference of the IEEE Computer and Communications Societies. Proceedings IEEE, vol. 3, pp. 2102-2111. IEEE, 2005.

\section{Author Details:}

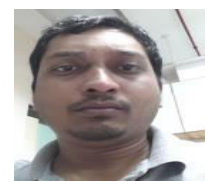

Koffka Khan received the M.Sc., and M.Phil. degrees from the University of the West Indies. $\mathrm{He}$ is currently a $\mathrm{PhD}$ student and has up-to-date, published numerous papers in journals \& proceedings of international repute. His research areas are computational intelligence, routing protocols, wireless communications, information security and adaptive streaming controllers.

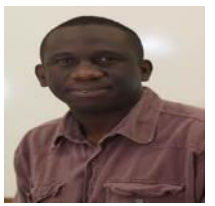

Wayne Goodridge is a Lecturer in the Department of Computing and Information Technology, The University of the West Indies, St. Augustine. He did is $\mathrm{PhD}$ at Dalhousie University and his research interest includes computer communications and security. 